\title{
Torque Ripple Improvement by Injecting High Voltage Pulses
}

\author{
Yang $\mathrm{Li}^{1, \mathrm{a}}$, Ming Feng ${ }^{1, \mathrm{~b}^{*}}$ \\ ${ }^{1}$ SME, University of Science and Technology Beijing, Haidian Distric, Beijing, PRC \\ aliyang_ustb@163.com, ${ }^{\text {b*}}$ mingfeng@me.ustb.edu.cn
}

Keywords: Torque ripple. Current rising-edge time. Leakage inductance. BLDC motor.

Abstract:This paper presents a new method of injection of high voltage pulses (IHVP) during commutation in order to shorten current's rising-edge time and reduce the torque ripple. A quantitative method for choosing the voltage magnitude and the pulse width is put forward with trapezoidal back EMF under square voltage wave condition. Its optimal discrete solutions and the principle of choosing the discrete pulse coefficient under PWM control are derived. Finally, the effect of the method is verified by experiments.

\section{Introduction}

With BLDC motor, a large leakage inductance is often caused by a full power output and numbers of winding turns, which lead to a severely abnormal current waveform and a serious torque ripple. Similar consequences can also be brought about by a much smaller leakage inductance when the motor speed is high enough, which signifies a shorter lead time. Moreover, different magnetization patterns lead to various odd harmonics of radial flux density on the rotor surface, which results in a great obstacle for the suppression of torque ripple.

Based on PWM-on modulation method, $\mathrm{Bu}$ [1] proposed a new method that duty cycle of the each phase is calculated by analyzing the current flow in commutation to suppress the commutation torque ripple. Zhang et al. [2] presented a new method for reducing the torque ripple in BLDC motors with a single current sensor by adding the step-down BUCK converter. Although the two methods are quite effective for commutation torque ripple reducing, they cost great amount of computation and lots of control resource. Li et al. [3] proposed a novel improved bipolar PWM scheme on the basis of the traditional bipolar PWM method, in which four power transistors are controlled simultaneously and the on-off states of two power transistors in one phase leg are opposite. This method is quite concise, but the effect is not obvious.

In this paper, a new method of injection of high voltage pulses (IHVP) is proposed, which is not only with properties of conciseness and accuracy but also much effective on the suppression of torque ripple.

\section{Theory of IHVP method}

Basic equations of IHVP method. For BLDC motors, the analytical solution of commutation currents meets the following equations ${ }^{[4]}$ 


$$
\left\{\begin{array}{l}
i_{a}(t)=-\frac{U+2 E}{3 R}+\left(I_{S}+\frac{U+2 E}{3 R}\right) e^{-\frac{1}{\tau} t} \\
i_{b}(t)=\frac{2 U-2 E}{3 R}+\frac{2 E-2 U}{3 R} e^{-\frac{1}{\tau} t} \\
i_{c}(t)=\frac{4 E-U}{3 R}+\left(I_{S}+\frac{4 E-U}{3 R}\right) e^{-\frac{1}{\tau} t}
\end{array}\right.
$$

where, $U$ is the phase voltage, $E$ is the back EMF of phase winding, $i(t)$ is the current in phase winding, $R$ is the phase resistance, $I s$ is the phase current under voltage $U$ before commutation, $\tau=L_{\sigma} / R$ is the electromagnetic time constant.

When current $i_{a}(t)$ in Eq. 1 decrease to 0 , the commutation process is considered to an end, the current falling-edge time $t_{u}$ is derived as

$$
t_{u}=-\tau \ln \left(\frac{U+2 E}{3 R I_{S}+U+2 E}\right)
$$

During commutation, the current rising-edge time is equal to the falling-edge time, thus $t_{u}$ is also the current rising-edge time. The shorter the current rising-edge time is, the more similar the current waveform is to a square wave, the smaller torque ripple is, and vice versa. From formula( 2 ), both deducing the electromagnetic time constant $\tau$ and raising the commutation voltage $U$ are advantageous to shorten current rising-edge time $t_{u}$. If pulses of high voltage were injected into the commutation circuit, which makes the conduction phase under a high voltage at initial time, the torque ripple could be effectively suppressed.

Parameters selection strategy of IHVP method. A BLDC motor with 2 poles and 24 slots is taken as an example. The motor parameters are shown in Table 1.

Table 1 Parameters of BLDC motor with 2P24S

\begin{tabular}{cc}
\hline Parameters & Magnitude \\
\hline Speed ( $\mathrm{r} / \mathrm{min})$ & 100,000 \\
Power ( $\mathrm{kW})$ & 10 \\
length ( $\mathrm{mm})$ & 65 \\
Stator outer diameter $(\mathrm{mm})$ & 80 \\
Rotor outer diameter ( mm ) & 27 \\
Rated current ( A ) & 48 \\
Time constant ( $\mathrm{s})$ & $2.17 \mathrm{e}-003$ \\
\hline
\end{tabular}

Voltage coefficient $K_{0}$ and pulse coefficient $K$ are defined as

$$
\begin{aligned}
& K_{0}=U / 2 E \\
& K=U^{\prime} / U
\end{aligned}
$$

For a certain back EMF, a bigger $K_{0}$ and $K$ mean a higher voltage $U$ and $U^{\prime}$, respectively. Substituting Eq. 3 and 4 into Eq.2, the rising-edge time is derived as

$$
t_{u}{ }^{\prime}=-\tau \ln \left(\frac{U^{\prime}+2 E}{3 R I_{S}+U^{\prime}+2 E}\right)=-\tau \ln \left(\frac{K U+2 E}{3 R I_{S}+K U+2 E}\right)
$$


and

$$
I_{S}=\frac{U-2 E}{2 R}\left(\frac{2 e^{\frac{10}{p n \tau}}-2}{2 e^{\frac{10}{p n \tau}}-1}\right)
$$

where the $p$ and $\mathrm{n}$ are the number of pole-pairs and the motor speed, respectively. Simultaneously solving Eqs. 3 to 6 yields

$$
t_{u}-t_{u}{ }^{\prime}=-\tau \ln \left\{\frac{\left(K_{0}+1\right)\left[\frac{3}{2}\left(K_{0}-1\right)\left(\frac{2 e^{\frac{10}{p n \tau}}-2}{2 e^{\frac{10}{p n \tau}}-1}\right)+K K_{0}+1\right]}{\left[\frac{3}{2}\left(K_{0}-1\right)\left(\frac{2 e^{\frac{10}{p n \tau}}-2}{2 e^{\frac{10}{p n \tau}}-1}\right)+K_{0}+1\right]\left(K K_{0}+1\right)}\right\}
$$

Equation 7 indicates that the saved rising-edge time is only relevant with parameter $K$ and $K_{0}$, when number of pole-pairs, motor speed and electromagnetic time constant are determined. The curve series of the saved rising-edge time vs various $K$ and $K_{0}$ is shown in Fig.1. From the figure, the larger $K_{0}$ or $K$ is, the more rising-edge time can be saved, which also means a greater improvement on torque ripple. However, both $K$ and $K O$ are limited by the largest available voltage $U_{\max }$. Furthermore, the ratio of the saved rising-edge time to its pulse coefficient $K$ represents the economy of pulse coefficient. On each curve that has a same $K_{0}$, there is a point represents the maximum value of the ratio. Connecting the points into a line, the recommended line of pulse coefficient is depicted in the figure. Therefore, the economy of pulse coefficient $K$ would be better if the pulse coefficient selected was closer to the recommended line.

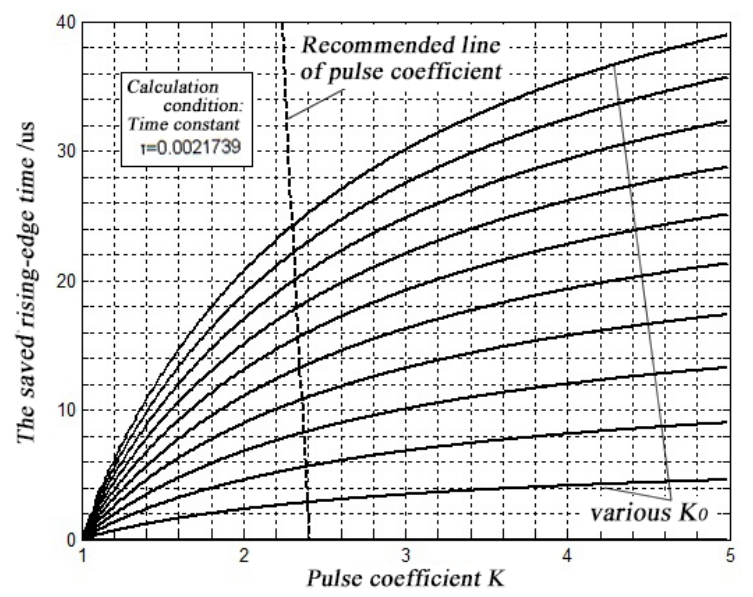

Fig. 1 The curves series of saved rising-edge time versus various $K_{0}$ and $K$

After the parameters selection, the voltage magnitude $U^{\prime}$ and the rising-edge time $t u^{\prime}$ can be quantitatively calculated by Eq.3 to 5. However, the rising-edge time $t u$ ' usually can't be chosen arbitrarily because it must be an integer times of the chopping period. If the chopping frequency is determined, the parameters in Eq.5 will be limited to some discrete values. Therefore, the continuous curves in Fig. 1 will be turned into discrete points. The chopping frequency of $100 \mathrm{kHz}$ is taken as an example. After the discretization of the curves, several discrete lines are obtained, as 
shown in Fig.2. The lines represent how many chopping periods the pulse width is equal to. Similarly, a pulse coefficient $\mathrm{K}$ can be economically chosen referencing the recommended line.

Moreover, the rising-edge time will not be fully reduced if the pulse coefficient is smaller than calculation. On the other hand, if the pulse coefficient is bigger, spikes will appear in the current waveform, which is not conducive to the suppression of the commutation torque ripple. Considering the chopping limitation, a smaller pulse coefficient $K$ is more appropriate if the two pulse coefficients have a similar saved rising-edge time.

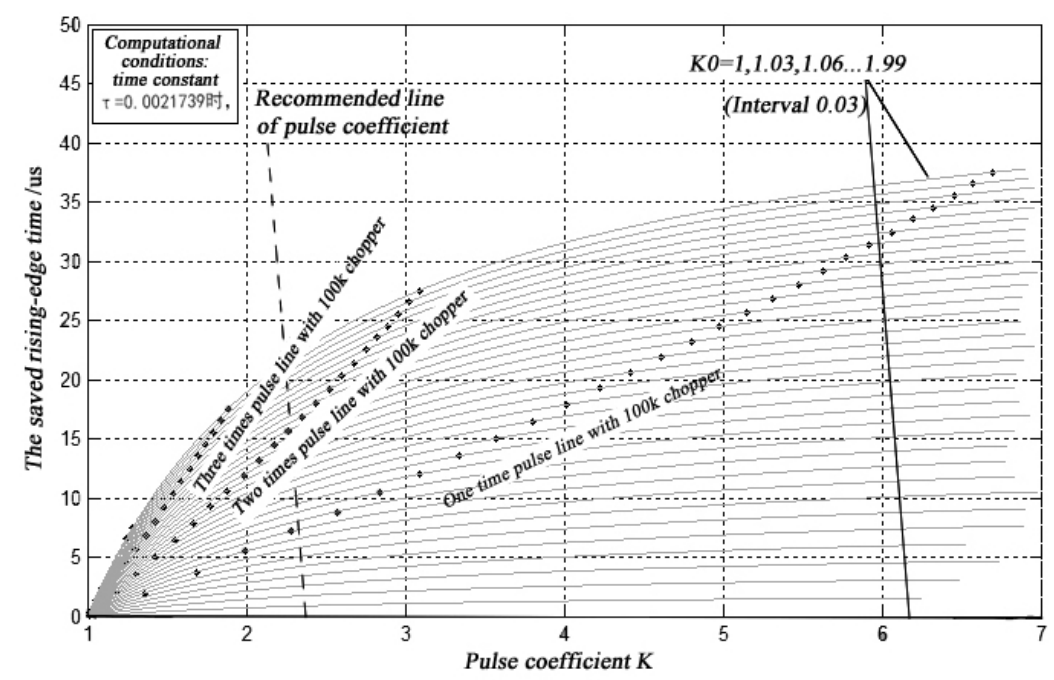

Fig. 2 The discrete saved rising-edge time versus various $K_{0}$ and $K$

\section{Simulation and experimental verification}

A simulation model of exciting IHVP into the three-phase bridge circuit was established with the motor mentioned in Table 1. The simulation results are shown in Fig. 3. The rising-edge time and the torque ripple are significantly decreased, which makes the current waveform much closer to a square wave.

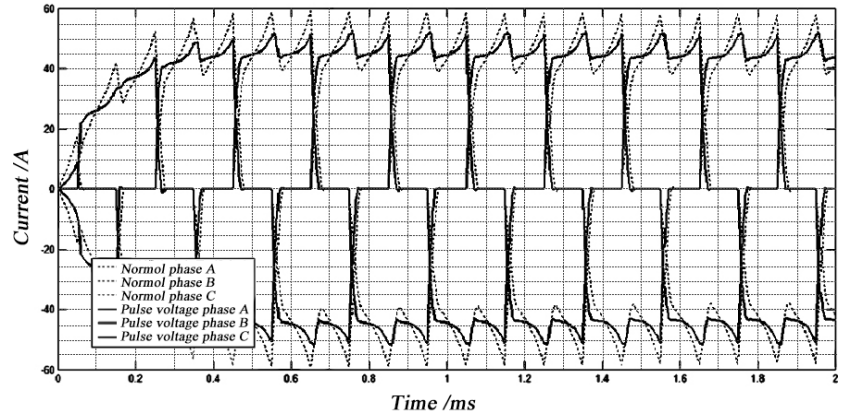

(a)Current

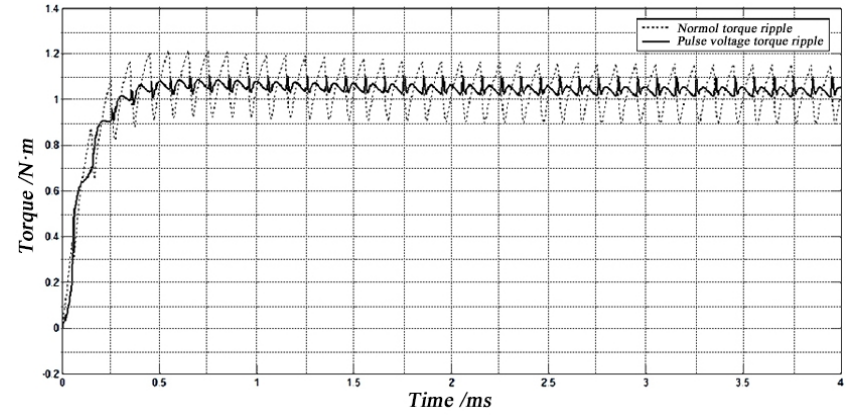

(b) Torque ripple

Fig.3 Theoretical currents and torque waveforms with and without IHVP method

A small power BLDC motor is used in experimental verification. Figure 4 shows the currents and torque ripple with and without IHVP method. With the IHVP method, the rising-edge time is evidently shortened and the current become stable. Since the voltage dead time has less effect on the current, torque ripple is effectively decreased, which contribute to the smoothness of motor running. 


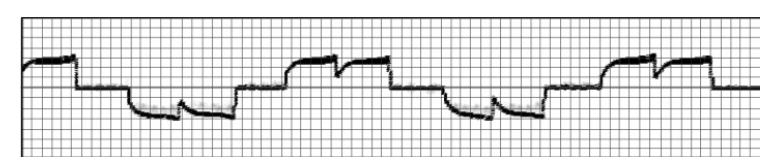

Experimental current waveform of BLDC motor with normal square wave control method

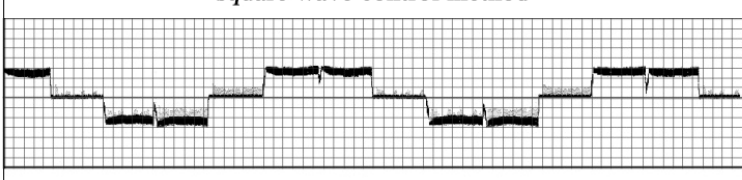

Experimental current waveform of BLDC motor with IPHV

(a) Current

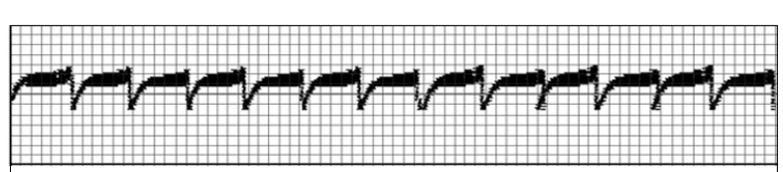

Experimental electromagnetic torque waveform of BLDC motor with normal square wave control method

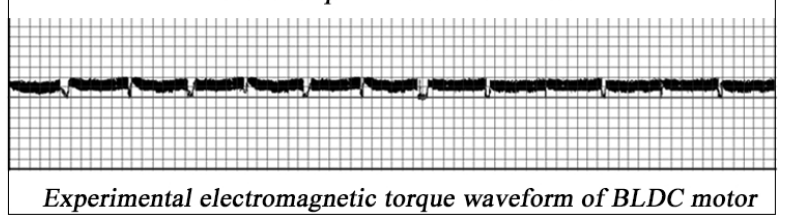

(b) Torque ripple

Fig.4 Experimental currents and torque waveforms with and without IHVP method

\section{Conclusions}

In this paper, we proposed a new method for solving BLDC motor's current distortion and commutation torque ripple, by which the current delay can be effectively shortened and its waveform is much closer to a square wave. The improvement of the current waveform and commutation torque ripple with the IHVP method is verified by both simulation and experiment. The main conclusions can be summarized as follows:

(1) A higher voltage in current rising-edge state results in a shorter current rising-edge time. The parameters $K_{0}$ and $K$ present the magnitude of original voltage and pulse voltage respectively, but both they are limited by the largest available voltage $U_{\max }$. The larger $K$ is, the more current rising-edge time can be saved.

(2) Limited by the chopping frequency, the rising-edge time $t u$ ' usually can't be chosen arbitrarily because it must be an integer times of the chopping period. Therefore, $K$ is turned into discrete instead of a continuous value.

(3) The ratio of the saved rising-edge time to its pulse coefficient $K$ represents the economy of pulse coefficient. The closer a pulse coefficient is to the line connected by the points of the maximum value of the ratio on each curve, the better the economy of pulse coefficient is.

(4) To prevent the current spike, a small pulse coefficient is appropriate for the similar control effect.

\section{References}

[1]Deming $\mathrm{Bu}$, Research on communication torque ripple of Permanent magnet BLDCM for electric vehicle In Chinese, D. Harbin Institute of Technology, 2013:7 - 8.

[2]Xiaofeng Zhang, Qongbo Hu, Zhengyu Lv, Torque ripple reduction in brushless DC motor drives using a Buck converter In Chinese, J. Transactions of China Electrotechnical Society, 2005, 020(009): 72 - 76, 81.

[3] Zicheng Li, Shanmei Cheng, Kai Cai, Yi Qin, Novel PWM scheme for reducing torque ripples in brushless DC motors In Chinese, J. Journal of Huazhong University of Science and Technology (Nature Science Edition), 2009, 037(011): 1-4.

[4] Li Yu, Hexiang Liu, Longfang Yi, The Circuit Model of Permanent Magnet Brushless DC Motor and Analysis of the Alternated Phase In Chinese, J. Micromotors, 2010, 043(010): 6 - 9. 